\title{
Interactive comment on "Hydrodynamic
} simulation of the effects of in-channel large woody debris on the flood hydrographs of a low mountain range creek, Ore Mountains, Germany" by Daniel \section{Rasche et al.}

\section{Daniel Scott (Referee)}

dan.scott@colostate.edu

Received and published: 31 May 2019

Please see the attached supplement for my review.

Please also note the supplement to this comment:

https://www.hydrol-earth-syst-sci-discuss.net/hess-2019-35/hess-2019-35-RC3- 
35, 2019.

HESSD

Interactive

comment

Printer-friendly version

Discussion paper

(c) (i) 